by Montgomery-Asberg Depression Rating Scale (MADRS) and Hamilton Anxiety Rating Scale (HAM-A).

Brain MRI including T1- weighted images, T2- weighted images and fluidattenuated inversion-recovery images (FLAIR) was done in $44(41,5 \%)$ BD patients.

Results: CD of mild to moderate severity were diagnosed in $82(77,4 \%)$ of BD patients. The mechanical memory $(50 \%)$ and attention deficit $(80,5 \%)$ were the most frequent manifestations of $\mathrm{CD}$, impairment of associative memory $(31,7 \%)$ and logical thinking $(36,6 \%)$ were less frequent. The presence of CD didn't depend on $\mathrm{BD}$ activity, severity and duration, as on patient's ethnicity, use of prednisone and immunosupressive agents. The frequency of neurological manifestations (headache, seizures, myelopathy, ataxia) did not differ significantly in patients with and without CD (28\% vs $32 \%, p=0,44)$. The patients with $C D$ were older $(34,3 \pm 1,07$ vs $29,0 \pm 2,14, p=0,006)$, more often had chronic/recurrent depressive disorders $(84,1 \%$ vs $50,0 \%, p=0,001)$ of moderate severity (MADRS $16,1 \pm 0,74$ vs $12,2 \pm 1,06, p=0,005)$, chronic stressful life events $(91,5 \%$ vs $62,5 \%, p=0,001)$ and multifocal subcortical parenchymal MRI changes $(57,6 \%$ vs $9 \%, p=0,005)$. Conclusions: the results have shown high rates of different $C D$ in $B D$ patients. $\mathrm{CD}$ were not associated with $\mathrm{BD}$ activity and presence of neurological symptoms. $\mathrm{CD}$ were related to the diagnoses of stress-related mild to moderate chronic depressive disorders and minor brain multifocal subcortical parenchymal MRI lesions.

Disclosure of Interest: None declared

DOI: 10.1136/annrheumdis-2017-eular.3741

\section{FRI0344 NOVEL BIOMARKERS OF SUBCLINICAL ENDOTHELIAL DYSFUNCTION IN BEHÇET'S SYNDROME: EVALUATION OF CROSS-SECTIONAL DISTENSIBILITY AND INTIMA-MEDIA-THICKNESS IN A HOSPITAL-BASED POPULATION}

R. Talarico ${ }^{1}$, C. Stagnaro ${ }^{1}$, S. Barsotti ${ }^{1}$, R. Neri ${ }^{1}$, C. Tani ${ }^{1}$, C. Baldini ${ }^{1}$, N. D Lascio $^{2}$, E. Bianchini ${ }^{2}$, A. Morales ${ }^{2}$, M. Mosca ${ }^{1} .{ }^{1}$ Department of Clinical and Experimental Medicine, University of Pisa, Rheumatology Unit; ${ }^{2} \mathrm{CNR}$, Institute of Clinical Physiology, Pisa, Italy

Background: Growing interest exists on the role of markers of subclinical cardiovascular disease as independent predictors of cardiovascular events. Poor data are available on the role of these markers as prognostic factors for Behçet's syndrome (BS)

Objectives: The primary aim was to explore Intima-Media-Thickness (IMT), mean arterial diameter and distensibility (DC) in a group of patients with BS, comparing these data with a healthy control group and a disease control group; the secondary aim was to correlate the vascular parameters with demographic/clinical profile.

Methods: Thirty BS patients (females:12;mean age \pm SD:43 \pm 10.5 ; mean disease duration $\pm S D: 13 \pm 5.8$ ) fulfilling the ISG criteria were prospective enrolled. Demographic data, level of disease activity, frequency of smokers, hypertension, family history of cardiovascular risk factors, body mass index (BMI) and current therapies were analysed. For each subject, ultrasound B-mode image sequences of right common carotid arteries were acquired and analysed by an automatic system (Carotid Studio,Quipu) for the measurement of IMT and mean arterial diameter. In addition, carotid pulse pressure (PP) was estimated by tonometry and DC coefficient was obtained. The systolic and diastolic carotid diameters were automatically measured on the distal wall of the common carotid artery, $1-2 \mathrm{~cm}$ beneath the bifurcation. Carotid diameter was calculated as the distance between media-adventitia interfaces. Cross-sectional DC was estimated through the variations in arterial cross-sectional area and blood pressure during systole. $D C$ was computed as $D C=\Delta A /\left(P P^{*} A\right)$ where $A$ is the diastolic lumen area, $\Delta \mathrm{A}$ is the stroke change in lumen area, and PP is the local pulse pressure.

Results: At time of evaluation, 4/17 patients presented active disease $(50 \%$ ocular involvement, $25 \%$ joint involvement, $25 \%$ gastro-enteric involvement; mean BS activity score 5). Mean IMT \pm SD value resulted of $0.57 \pm 0.81$, mean arterial diameter $\pm S D$ value was $6.879 \pm 0.81$ and mean $D C \pm S D 27.3 \pm 14.34$. All the vascular parameters considered were significant correlated with BMI, while only IMT and DC were also significant correlated with arterial hypertension. Using a correction analysis for age and sex, we found significant correlations between mean arterial diameter and disease activity and between DC and disease duration. These data resulted significantly different compared to healthy control and a disease control groups, in terms of smaller arterial diameter and higher DC. Conclusions: Our data have shown that there is a consistent influence of disease activity and duration of disease on mean arterial diameter and DC, respectively. Thus, it is desirable that future pharmacological researches on BS target on this issue.

Acknowledgements:

Seyahi E, Ugurlu S, Cumali R, Balci H, Ozdemir O, Melikoglu M, Hatemi G,

Fresko I, Hamuryudan V, Yurdakul S, Yazici H. Atherosclerosis in Behçet's

Syndrome. Semin Arthritis Rheum. 2008 Aug;38(1):1-12.

Seyahi E, Ugurlu S, Cumali R, Balci H, Ozdemir O, Melikoglu M, Hatemi G,Fresko

I, Hamuryudan V, Yurdakul S, Yazici H. Atherosclerosis in Behçet'sSyndrome.

Semin Arthritis Rheum. 2008 Aug;38(1):1-12.

Disclosure of Interest: None declared

DOI: 10.1136/annrheumdis-2017-eular.5907

\section{FRI0345 CLINICAL IMPACT OF ALPHA-1-ANTITRYPSIN DEFICIENCY IN GRANULOMATOSIS WITH POLYANGIITIS}

S. Deshayes ${ }^{1}$, A. Aouba ${ }^{1}$, F. Grandhomme ${ }^{2}$, K. Kathy ${ }^{3}$, D. Mariotte ${ }^{3}$, ${ }_{\text {J. Boutemy }}{ }^{1}$, G. Maigné ${ }^{1}$, C. Brière-Bellier ${ }^{4}$, C. Delmas $^{1}$, B. Bienvenu ${ }^{1}$ N. Martin Silva ${ }^{1} .{ }^{1}$ Department of Internal Medicine; ${ }^{2}$ Department of Biochemistry; ${ }^{3}$ Department of Immunology, CHU Côte de Nacre, Caen; ${ }^{4}$ Department of Infectious Diseases, CH Mémorial, Saint-Lô, France

Background: Deficiency in $\alpha$-1-antitrypsin (AAT), which is the main proteinase 3 (PR3) inhibitor, is now recognized as a pathogenic factor in some cases of anti-PR3 anti-neutrophil cytoplasmic antibodies (ANCA) related granulomatosis with polyangiitis (GPA). However, the clinical impact of AAT deficiency remains poorly established in this setting.

Objectives: The purpose of our study was to describe the clinical phenotypes and outcomes of anti-PR3 GPA patients according to their AAT status.

Methods: A retrospective monocentric study carried out in Caen University Hospital led to identify anti-PR3 GPA patients, from 09/21/2011 to 06/10/2016. AAT dosage and phenotype (isoelectric focusing in agarose gel) were performed for all patients. Categorical variables were reported as percentages and compared using $\mathrm{Chi}^{2}$ or Fisher's tests according to expected frequencies. Continuous variables were expressed as means and analysed using Student's t-test. Associations between survival, renal survival or relapse-free survival, and AAT phenotype were evaluated by the log-rank test. A p-value $<0.05$ was considered to be statistically significant.

Results: Among the 72 identified anti-PR3 GPA patients, 40 (56\%) were male. Median age at diagnosis was 60.5 years old. Patients mainly had constitutional symptoms $(51,71 \%)$, pulmonary $(52,72 \%)$, ear, nose or throat (ENT) $(49,68 \%)$ rheumatologic $(45,63 \%)$, and renal $(44,61 \%)$ involvements. Median initial BVAS score was 38 (maximum score: 63 ). Twelve $(17 \%)$ deaths and $33(46 \%)$ relapsing patients were noted (median follow-up: 55 months). Forty-eight $(67 \%)$ patients had MM phenotype, 10 (14\%) MZ phenotype, 8 (11\%) MS phenotype, $3(4 \%)$ M-variant phenotype, $2(3 \%)$ ZZ phenotype and 1 (1\%) ZS phenotype. Allele frequencies of $M, Z$ and $S$ allele were 81,10 and $6 \%$, respectively. The whole patient cohort had the same immunosuppressant drug regimen.

Tab;e 1

\begin{tabular}{lcccccc}
\hline & $\begin{array}{c}Z \\
\text { carriers } \\
(\mathrm{n}=13)\end{array}$ & $\begin{array}{c}\text { Non-Z } \\
\text { carriers } \\
(\mathrm{n}=59)\end{array}$ & $\begin{array}{l}\mathrm{p} \text {-value } \\
\text { (n) }\end{array}$ & $\begin{array}{c}\mathrm{Z} \text { or S } \\
\text { carriers } \\
(\mathrm{n}=21)\end{array}$ & $\begin{array}{c}\text { Non Z or S } \\
\text { carriers } \\
(\mathrm{n}=51)\end{array}$ & $\mathrm{p}$-value \\
\hline Initial BVAS (median) & 18 & 18 & 0.91 & 18 & 18 & 0.71 \\
Death (\%) & 15 & 17 & 1 & 14 & 18 & 1 \\
Age at diagnosis (years, median) & 61 & 60 & 0.91 & 61 & 59 & 0.62 \\
Relapse (\%) & 38 & 47 & 0.56 & 43 & 47 & 0.75 \\
Intra-alveolar hemorrhage (\%) & 47 & 14 & $\mathbf{0 . 0 0 4}$ & 43 & 12 & $\mathbf{0 . 0 0 9}$ \\
ENT (\%) & 92 & 63 & $\mathbf{0 . 0 5}$ & 67 & 69 & 0.88 \\
AAT level (g/L, median) & 1.07 & 1.65 & $<\mathbf{0 . 0 0 1}$ & 1.11 & 1.65 & $<\mathbf{0 . 0 0 1}$ \\
\hline
\end{tabular}

Only intra-alveolar hemorrhage $(\mathrm{IAH})$, that was more frequent in $\mathrm{Z}$ or $\mathrm{S}$ deficient allele patients, and ENT involvement, that was more frequent in those with $Z$ allele, were significantly associated with AAT deficiency. Among the 13 patients carrying $Z$ allele, 9 had normal AAT level, including 6 without any biologic inflammatory parameter at the time of AAT dosage. Global survival, renal survival or relapse-free survival were identical between $\mathbf{Z}$ carriers compared to non-Z carriers (respectively $0.77,0.75$ and 1 ), and between $Z$ or $S$ carriers compared to those without (respectively $0.91,0.44$ and 0.32 ).

Conclusions: This study confirms the epidemiological association of anti-PR3 GPA with AAT deficiency, and find an interesting higher risk of IAH in this setting. The identical prognosis of both subgroups should be related to other therapeutic added in $\mathrm{IAH}$, including plasma exchanges. Prospective studies are required to specify these data and to assess the need for replacement therapy in AAT deficient patients.

Disclosure of Interest: None declared

DOI: 10.1136/annrheumdis-2017-eular.4500

\section{FRI0346 RENAL INVOLVEMENT IN GRANULOMATOSIS WITH POLYANGIITIS}

N. Bulanov ${ }^{1}$, E. Makarov ${ }^{2}$, E. Kuznetsova ${ }^{1}$, M. Bulanova ${ }^{3}$, P. Novikov ${ }^{1}$ S. Moiseev ${ }^{1}$. ${ }^{1}$ Sechenov First Moscow State Medical University; ${ }^{2}$ Lomonosov Moscow State University, Moscow; ${ }^{3}$ Vladimir Regional Clinical Hospital, Vladimir, Russian Federation

Objectives: We assessed the frequency, clinical features and prognosis of renal involvement in granulomatosis with polyangiitis (GPA) and current treatment approaches.

Methods: We performed a retrospective analysis of 234 patients with GPA diagnosed according to Chapel-Hill Consensus Conference 2012 classification, 81 male and 153 female, aged $53(41 ; 62)$ years. 54 patients $(23.1 \%)$ had localized GPA. 174 (74.4\%) were ANCA positive. Median follow up was 61.5 (32; $105)$ months. 103 patients $(44 \%)$ had a history of renal involvement. Frequencies of proteinuria, hematuria, hypertension, rapidly progressive glomerulonephritis (RPGN), acute kidney injury (AKI) and chronic kidney disease (CKD) grades were analyzed.

Results: In 25 patients (24.3\% of 103 ) renal involvement developed at disease 
onset, the other 78 patients $(75.7 \%)$ developed glomerulonephritis after a median of $3(1 ; 12)$ months. Hematuria was present in $94(91.3 \%)$, proteinuria in 85 patients $(82.5 \%)$, nephrotic syndrome in 11 patients $(10.7 \%)$, hypertension in 31 patients (30.1\%). Rapidly progressive glomerulonephritis (RPGN) defined by the doubling of serum creatinine within $\leq 3$ months developed in 29 patients $(28.2 \%$ of 103$)$. Myeloperoxidase-ANCA-positive patients $(n=12)$ developed RPGN significantly more often than proteinase-3-ANCA-positive patients $(n=78): 7(58.3 \%)$ vs 22 $(28.2 \%)(p=0.0499) .11(10.7 \%)$ patients developed AKI, stage 3 being the most common (in 8 patients). 40 (38.8\%) patients exhibited indolent renal disease course, i.e. without worsening of renal function. By the end of the follow-up, 33 $(32.0 \%)$ patients were diagnosed with CKD grade $3 b-5$, among them 11 patients (10.7\%) developed end-stage renal disease (ESRD). 6 patients (5.8\%) died. As the first line induction therapy all patients received corticosteroids in combination with cyclophosphamide in 93 patients $(90.3 \%)$, rituximab (RTX) in 3 patients $(2.9 \%)$, mycophenolate mofetil in 7 patients $(6.8 \%)$. RTX was used for remission induction in $27(26.2 \%)$ patients due to refractory or recurrent disease. During follow-up, 74 patients $(71.8 \%)$ developed one or more severe relapses with a relapse rate of 0.27 per patient-year. $50(48.5 \%)$ patients had renal relapses (0.096 per patient-year).

Conclusions: Prevalence of renal involvement in the studied group was lower than expected, most likely because of a large proportion of patients with localized GPA. Our study showed that RPGN was not the most common feature of renal involvement. Almost $40 \%$ of patients developed indolent course of kidney disease. Nevertheless one third of patients developed CKD G3B or worse by the end of the follow up. Despite effective induction therapy with high-dose corticosteroids, cytotoxic agents and/or RTX relapses, both renal and extrarenal, are frequent.

Disclosure of Interest: None declared

DOI: 10.1136/annrheumdis-2017-eular.5104

\section{FRI0347 RHEUMATOID FACTOR IS CORRELATED WITH DISEASE ACTIVITY AND INFLAMMATOTY MARKERS IN ANTINEUTROPHIL CYTOPLASMIC ANTIBOTY-ASSOCIATED VASCULITIS}

S. Watanabe, T. Gono, K. Nishina, N. Sugitani, E. Watanabe, H. Yabe, C. Terai. Rheumatology, Jichi Medical University, Saitama Medical Center, Saitama, Japan

Background: Raised levels of serum rheumatoid factors (RFs) of different immunoglobulin classes had been reported in a high proportion of patients with rheumatoid vasculitis. RF used to be studied and was shown elevated in some forms of vasculitides. Patients with antineutrophil cytoplasmic antibody (ANCA)-associated vasculitis (AAV) are often positive for RF. However, the clinical significance of RF has been seldom examined in AAV.

Objectives: The aim of this study was to investigate association between the presence of RF and clinical features and outcomes in AAV.

Methods: Eighty-one patients were diagnosed with AAV from 2006 to 2015 in our hospital. Among 81 patients, forty-seven patients $(17$ males, median age 67 years) who were not complicated with rheumatoid arthritis and in whom RF was measured before the treatment, were studied, retrospectively. Patients were classified using the European Medicines Agency vasculitis classification algorithm. AAV included eosinophilic granulomatosis with polyangitis $(n=10)$, granulomatosis with polyangitis $(n=14)$, microscopic polyangitis $(n=16)$ and unclassifiable vasculitis (UV) ( $n=7)$. Patients with UV with MPO-ANCA or PR3-ANCA were included in this study. IgM-RF was measured using a latex agglutination assay. Disease activity was assessed with Birmingham vasculitis activity score (BVAS). IgM-RF, C-reactive protein (CRP), erythrocyte sedimentation rate (ESR), serum ferritin, IgG, IgM, IgA, MPO-ANCA and PR3-ANCA were obtained from hospital records. Clinical manifestations between RF-positive subset $(n=29)$ and RF-negative subset $(n=18)$ were analyzed using Fisher's exact and Wilcoxon rank sum tests. Correlation coefficients were established with Spearman's correlation coefficient. Data were shown as medians (interquartile range).

Results: BVAS was higher (14 (12-22) vs $12(6-16), P=0.026)$ in the RF-positive subset than the RF-negative subset. CRP and ESR were higher $(P=0.020$ and 0.007 , respectively) in the RF-positive patients. IgM-RF titers significantly correlated with BVAS $(r=0.50, P=0.0004)$. In addition, CRP, ESR, IgM and IgG also had a significant correlation with IgM-RF titers. The frequency of initiation of dialysis therapy (14\% vs $6 \%)$, usage of mechanical ventilation (14\% vs $0 \%)$ and mortality ( $10 \%$ vs $0 \%$ ) were higher in the RF-positive subset than in the RF-negative subset although no significant differences were shown.

Conclusions: In AAV, IgM-RF titers are significantly correlated with disease activity and inflammatory markers. Presence of RF could be a poor prognostic factor in patients with AAV.

References:

[1] Watts R, Lane S, Hanslik T, et al. Development and validation of a consensus methodology for the classification of the ANCA-associated vasculitides and polyarteritis nodosa for epidemiological studies. Ann Rheum Dis 2007;66:222227.

[2] Westedt ML, Herbrink P, Molenaar JL, et al., Rheumatoid factors in rheumatoid arthritis and vasculitis. Rheumatol Int 1985;5:209-14.

[3] Kronbichler A, Kerschbaum J, Gründlinger G, et al. Evaluation and validation of biomarkers in granulomatosis with polyangiitis and microscopic polyangiitis. Nephrol Dial Transplant 2016;31(6):930-6.

Disclosure of Interest: None declared
DOI: 10.1136/annrheumdis-2017-eular.4555

\section{FRI0348 ASSOCIATED INFLAMMATORY SYNDROMES IN TAKAYASU'S ARTERITIS: MANY FACES OF A DISEASE}

S.N. Esatoglu ${ }^{1}$, A.M. Celik ${ }^{2}$, D. Ucar ${ }^{3}$, A.F. Celik ${ }^{4}$, S. Ugurlu ${ }^{1}$, G. Hatemi ${ }^{1}$, M. Melikoglu ${ }^{1}$, I. Fresko ${ }^{1}$, V. Hamuryudan ${ }^{1}$, H. Ozdogan ${ }^{1}$, S. Yurdakul ${ }^{1}$, H. Yazici ${ }^{1}$, E. Seyahi ${ }^{1}$. ${ }^{1}$ Istanbul University, Cerrahpasa Medical School, Department of Internal Medicine, Division of Rheumatology; ${ }^{2}$ Istanbul University Cerrahpasa Medical School, Department of Internal Medicine; ${ }^{3}$ Istanbul University, Cerrahpasa Medical School, Department of Ophthalmology; ${ }^{4}$ Istanbul University, Cerrahpasa Medical School, Department of Internal Medicine, Division of Gastroenterology, Istanbul, Turkey

Background: Case reports and series suggest that Takayasu's arteritis (TA) can co-exist with various inflammatory disorders.

Objectives: We conducted a formal study to look specifically at the frequency of inflammatory disorders and symptoms in a large cohort of TA followed by a single tertiary center.

Methods: There were 238 patients registered with a diagnosis of TA. Of these, 19 died, 18 were lost to follow-up and 3 did not wish to response our questionnaire. The remaining 198 patients were called back at the outpatient clinic. A standardized form sought whether the patient was also diagnosed as inflammatory bowel disease (IBD), ankylosing spondylitis (AS), Behçet's syndrome (BS), amyloidosis, uveitis, rheumatoid arthritis (RA), systemic lupus erythematosus (SLE), systemic sclerosis (pSS), Sjögren syndrome, psoriatic arthritis, inflammatory myositis, small vessel vasculitis, autoimmune/demyelinating or any other inflammatory disorder.

Results: Overall, 198 (175 F/ 23 M) patients were studied. The mean age at the time of TA diagnosis was $34 \pm 12$ years. Subclavian artery was the most common involved artery (84\%), followed by common carotids $(78 \%)$ and aorta (65\%). Currently, while $25(16 \%)$ patients were off treatment, $72(47 \%)$ patients were using glucocorticoids, 47 (31\%) azathioprine, $32(21 \%)$ methotrexate and $44(29 \%)$ biological agents.

We identified in total $37(19 \%)$ patients with inflammatory diseases (IBD: $n=12$; AS: $n=15$; and BS: $n=10$ ). Table shows their demographic characteristics. Among the remaining 161 patients, the most frequent feature was inflammatory back pain $(36 \%)$, followed by recurrent oral ulcers $(15 \%)$, erythema nodosum $(11 \%)$, arthritis $(10 \%)$, papulopustular lesions $(7 \%)$, uveitis $(4 \%)$, and genital ulcer $(1 \%)$. It was noted that inflammatory back was mostly located on the dorsal area. Regarding autoimmune diseases, we also observed RA $(n=3)$, psoriasis $(n=2)$, autoimmune hepatitis $(n=2)$, SS $(n=1)$ and SSc $(n=1)$.

Table 1. Demographic features of 37 TA patients with inflammatory bowel disease (IBD), ankylosing spondylitis (AS) or Behçet's syndrome (BS)

\begin{tabular}{lcccc}
\hline Concomitant disease & F/M & $\begin{array}{c}\text { Mean age at TA } \\
\text { diagnosis (SD) }\end{array}$ & $\begin{array}{c}\text { Mean age at concomitant } \\
\text { disease (SD) }\end{array}$ & $\begin{array}{c}\text { Time of TA diagnosis } \\
\text { in relation to } \\
\text { concomitant disease }\end{array}$ \\
\hline AS $(n=15)$ & $13 / 2$ & $31 \pm 8$ & $27 \pm 11$ & $\begin{array}{l}\text { Simultaneous }(n=3) \\
\text { TA preceded }(n=7) \\
\text { AS preceded }(n=5) \\
\text { Simultaneous }(n=9) \\
\text { TA preceded }(n=1) \\
\text { IBD preceded }(n=2) \\
\text { IBD }(n=12)\end{array}$ \\
& $11 / 1$ & $33 \pm 9$ & $31 \pm 9$ & $\begin{array}{l}\text { Simultaneous }(n=5) \\
\text { TA preceded }(n=1) \\
\text { BS preceded }(n=4)\end{array}$ \\
BS $(n=10)$ & $7 / 3$ & $35 \pm 13$ & $32 \pm 13$ &
\end{tabular}

Conclusions: TA does co-occur with IBD, AS or BS in about $1 / 5$ of the patients, at least in a hospital setting and without a clear temporal pattern. This could be due to the close association of TA with MHC class-1 diseases. In addition, the high prevalence of inflammatory back pain in the dorsal spine in TA needs further scrutiny.

Disclosure of Interest: None declared

DOI: 10.1136/annrheumdis-2017-eular.6276

\section{FRI0349 THE SNP RS6871626 LOCATED IN IL12B REGION MAY INFLUENCE ON VASCULAR LESIONS OF TAKAYASU ARTERITIS}

T. Nakajima ${ }^{1}, \mathrm{H}$. Yoshifuji ${ }^{1}, \mathrm{C}$. Terao $^{2}, \mathrm{~K}$ Murakami $^{1}, \mathrm{R} . \mathrm{Nakashima}^{1}$, Y. Imura ${ }^{1}, \mathrm{~K}$. Ohmura ${ }^{1}$, T. Mimori ${ }^{1}$. ${ }^{1}$ Department of Rheumatology and Clinical Immunology, Graduate School of Medicine, Kyoto University; ${ }^{2}$ Center for Genomic Medicine, Kyoto University, Kyoto, Japan

Background: Takayasu arteritis (TAK) is a type of large vessel vasculitis, which affects aorta and its main branches. We previously found a single nucleotide polymorphism (SNP), rs6871626 located in IL12B region, as a susceptible gene to TAK and reported that the risk allele at the SNP is associated with the risk of aortic regurgitation $(A R)^{1)}$. However, there have been no studies on the association of the SNP with the organ involvements other than AR in TAK patients.

Objectives: To investigate the association of the SNP with arterial and organ involvements except for AR.

Methods: We examined the medical records of 85 patients with TAK, stratified 